\title{
Correction to: A Clinical Approach to Diagnosing Trigger Wrist
}

\author{
M. Arumugam ${ }^{1}$ (I) H. Sallehuddin ${ }^{2} \cdot$ F. M. N. Rashdeen ${ }^{3}$
}

Published online: 7 December 2020

(c) Indian Orthopaedics Association 2020

\section{Correction to: Indian Journal of Orthopaedics https://doi.org/10.1007/s43465-020-00248-7}

The original version of this article unfortunately contained a mistake. The abstract was incorrect; the corrected abstract is given below. The original article has been corrected.

\begin{abstract}
Background Trigger wrist is a relatively unusual condition, produced by wrist or finger motion. The various causes of trigger wrist can originate from flexor tendon, extensor tendon, bones, or tumour. A proper clinical approach is required to diagnose and manage patients with trigger wrist
\end{abstract}

Methods A keyword search was performed across Google Scholar and PubMed. Articles describing trigger wrist conditions were analysed. Based on the information obtain from the articles, the clinical manifestations and approach to diagnosing the cause of trigger wrist are discussed.

Results A detailed history alone may lead to a reasonably accurate diagnosis. Patients can present with trigger wrist occurring during movement of the fingers or with wrist movements. Presence of tenderness around A1 pulley suggests trigger finger. Absence of tenderness over the A1 pulley may suggest trigger wrist. The wrist should be examined for any swelling or malunion around the wrist joint;

The original article can be found online at https://doi.org/10.1007/ s43465-020-00248-7.

M. Arumugam

manohar.arumugam@gmail.com

1 Department of Orthopaedic Surgery, Faculty of Medicine and Health Science, Universiti Putra Malaysia, Serdang, Selangor, Malaysia

2 Department of Orthopaedics and Traumatology, Hospital Sultanah Bahiyah, Alor Setar, Kedah, Malaysia

3 Department of Orthopaedics and Traumatology, Hospital Kuala Lumpur, Kuala Lumpur, Malaysia palpate for any bony prominence, clicking, or crepitus with the movement of the wrist. Examination for the presence of carpal tunnel syndrome should be performed. A simple radiograph of the wrist joint is needed to see any possible bony pathology such as malunion, instability, or arthritis of the carpal bone. For soft-tissue assessment, ultrasound would be a good choice and can be done during finger or wrist movement. MRI is useful for further assessment of space occupying lesion within the carpal tunnel and is useful for surgical planning. Nerve conduction study is indicated for patients with median nerve compression symptoms. During the initial stage, the patient should be advised for activity modification to reduce the wrist and finger movements. Surgical treatment will depend on the causative factor. Surgery done under local anaesthesia has the advantage of reconfirming with the patient, resolution of triggering during surgery by asking the patient to actively move the fingers or wrist.

Conclusions Trigger wrist is a relatively rare condition compared with trigger finger, which is the most common disorder of the hand. To avoid inadequate and ineffective treatment of patients with trigger wrist, careful examination and proper diagnosis are vital.

Publisher's Note Springer Nature remains neutral with regard to jurisdictional claims in published maps and institutional affiliations. 